\title{
DIVERSITY IN DESIGN: ADDRESSING THE BLACK AWARDING GAP
}

\author{
Gary UNDERWOOD ${ }^{1}$ and Franziska CONRAD ${ }^{2}$ \\ ${ }^{1}$ Bournemouth University \\ ${ }^{2}$ Arts University Bournemouth
}

\begin{abstract}
Recent international events have focused attention on the issues of systemic racism and colonialism within institutions, and a spotlight has been shone on UK universities' attempts to evolve in creating secure and inclusive futures in the face of societal change. While access to University education for Black students has improved significantly in the UK over the past decade, there remains a major discrepancy in success rates at degree level. Students with an African or Caribbean heritage are considerably less likely to obtain good honours (first or 2:1) in their degree classification than any other ethnic group and are also less likely to maintain their course through to completion. While differences in entry qualifications may play a part, the larger part of this awarding and retention gap must be due to other factors.

Drawing on an overview of the initiatives currently being proposed by the UK's top creative universities to tackle the awarding gap, this paper uses interviews with past and present Black students from art and design courses at Arts University Bournemouth (AUB) and Bournemouth University (BU) to explore a range of issues - including economic and cultural barriers, institutional bias, educational content, communication issues, and direct and indirect racism. Some of the proposed initiatives for tackling the problem are also discussed, and while the responses highlight the underlying complexity which is causing the slow progress in closing the awarding gap for Black students in higher education, the desire to be better understood and supported as individuals by lecturers and peers, with greater opportunity for open and frank dialogue, was paramount.
\end{abstract}

Keywords: Black, BAME, attainment, diversity, awarding gap

\section{INTRODUCTION}

During 2020 international events focused attention on the issues of systemic racism and colonialism in the UK and across the world. The deaths of Breonna Taylor and George Floyd at the hands of US police officers, followed by global protests and the eruption of the Black Lives Matter movement sent shockwaves through every sector of society, including education. Universities play a pivotal role in responding to societal change and creating a secure and inclusive future for the design industry. While most UK universities have recognized the issues surrounding Black, Asian and Minority Ethnic (BAME) student retention and attainment for many years and have facilitated a range of initiatives to combat these problems, 2020 has seen more widespread scrutiny and greater urgency in HE's efforts to address its treatment of Black students and staff [1].

Over the past decade access to University education for Black ethnic groups has improved significantly in the UK. According to the admissions service UCAS, in England the proportion of Black 18-year-olds entering higher education rose from $28 \%$ in 2010 to $45 \%$ in 2019. This represents the biggest increase of any ethnic group, and the entry rate is now well above the national average rate of $35 \%$. In 2019 English undergraduate courses welcomed almost 51,000 Black students [2].

However, there remains a very significant discrepancy in success rates at degree level for students with a Black heritage. Figures from the UK independent regulator Office for Students (OfS) indicate that Black students are around $10 \%$ less likely than any other ethnic group to be awarded a first or upper second classification, as former Education Secretary Damien Hinds acknowledged in 2020:

What we were absolutely aware of was this gap in attainment at firsts and 2:1s. The Office for Students is focused on this, trying to identify what the blockage is. [3] 
Part of this discrepancy can be statistically explained by differences in age and entry qualifications: Black Caribbean students in particular are, on average, older when entering Higher Education (HE), and are more likely to have progressed via college or vocational qualifications [4]. However, the OfS estimates that there still remains an 'unexplained' gap of 17 percentage points between Black and White graduates, based on 2016/17 figures [5]. In addition, the OfS also reports that Black students are more likely than any other ethnic group to withdraw early from Higher Education, with a non-continuation rate of $15.0 \%$. This compares to a figure of $8.7 \%$ for White students [6]. Although Connor et al. [4] argues that that this discrepancy reduces considerably once a number of key factors are taken into account - such as age, subject, entry qualifications and gender - the issue still remains and has steadily worsened since 2010 .

\section{THE SCOPE OF THIS PAPER}

From their personal experience teaching at Bournemouth University (BU) and Arts University Bournemouth (AUB), and from previous research looking at BAME issues in design education, the authors had been aware that progress on reducing the awarding gap had been limited, despite awareness of this issue for many years. In order to try and gain further insight into this, the authors conducted a review of the latest measures and initiatives put in place by a number of universities that were specifically targeted at reducing the Black awarding gap. This was followed by discussions with several Black alumni of BU and AUB to ask them about some of the issues they faced while at university, and to try and gain their views on the validity of the planned approaches.

While most universities refer to this issue as the 'attainment gap', the authors have chosen to use the phrase 'awarding gap'. This is in line with UCL's BAME Awarding Gap Project, which rejects the term attainment as 'it implies that the responsibility of inequality in attainment lies with the student's themselves' [7].

\section{DETERMINING THE TOPICS FOR DISCUSSION}

In deciding which universities to look at with regard to initiatives, the authors were keen to focus on institutions with a strong design profile. For that reason, the top twenty universities in the 2020 Guardian university league table for design and crafts were targeted. Any measures targeted at the Black or BAME awarding gap appearing in university Access and Participation Plans and publicly available Diversity and Inclusion documentation was recorded. In addition, wherever an institution declared a link to any resources such as the UCL Toolkit, the UUK/NUS \#Closing the Gap Report, or the PReSS Project, measures contained within these initiatives was also noted. A total of 70 distinct measures were listed, and these were grouped into 16 broad areas. A bar chart was created to show how many universities included at least one of the measures contained within each one of these areas (Figure 1).

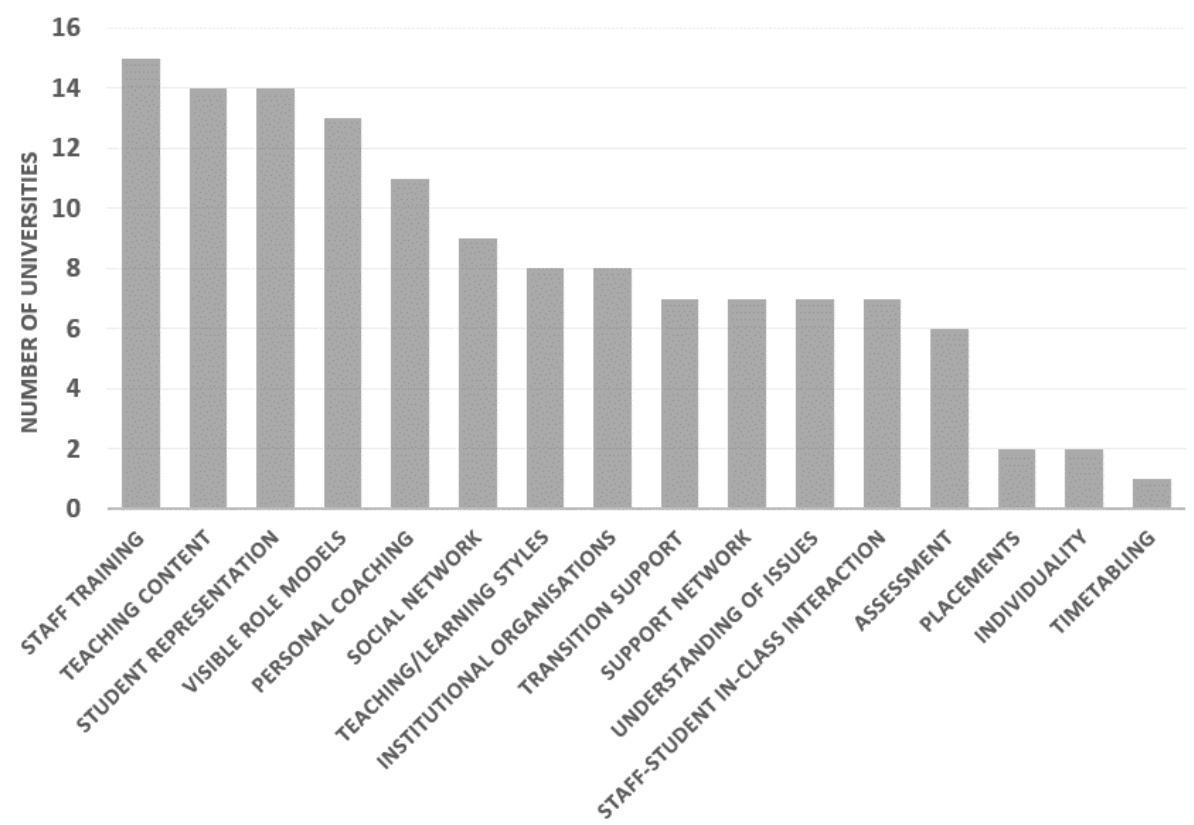

Figure 1. Frequency of areas mentioned 


\subsection{Other issues}

While it is commonly agreed that the reasons behind reduced Black success rates are many and varied, the research conducted by Connor et al. for the UK Government [3] pinpointed the most frequently reported issues that BAME students felt had affected their academic performance as:

- financial difficulties

- problems with balancing part-time work and study

- $\quad$ problems with facilities and getting support from staff

The research also reported that Black African students were the most likely ethnic group to experience problems that they felt had affected their academic results, and suggested that the following issues are likely to also have an effect on BAME student outcomes:

- difficulties in adjusting to life within a group with different social backgrounds

- $\quad$ issues with establishing relationships and getting support from tutors (Black students specifically)

- lack of confidence

- difficulties in transition between school and HE

While it can be argued that the focus of this research places the onus on the students themselves, rather than on any deficiencies in the content or culture of the teaching establishments, it is interesting to note that many of the issues described above were not strongly addressed by the university initiatives. The authors therefore felt that it would be useful to discuss these issues with the participants.

It was also noted that all of the universities consistently use the terms BAME or BME within their initiatives, despite the fact that in at least two cases the local Student Unions professed misgivings about the use of the terms. The Secretary of State for Health and Social Care Matt Hancock drew criticism from many quarters in 2020 by answering a question on the lack of Black representation in the UK Government by referencing the two 'Black and ethnic minority' members of the UK cabinet - both of whom are of South Asian heritage - and the 'lazy homogenisation of all non-white groups' [8] has become increasingly under fire, particularly amongst some Black groups. It was therefore decided that questions on terminology would be included.

\section{THE RESPONSES}

The authors contacted 11 Black alumni in total, of whom six agreed to participate in the research; this comprised of five product design graduates from BU and one fine art graduate from AUB. The years of study covered 2009 to 2020 and ages ranged from 24 to 30. Five of the participants were female - four with a Black African background, one with a Black Caribbean background - and the one male participant had a Black African background.

Prior to the discussions, a questionnaire was distributed to collect some background information. This determined that almost all of the participants had a term-time job while studying at university, including during the final year, and this could be for up to 24 hours per week. While most of the participants had not experienced direct racial abuse while at university, half felt that they had experienced indirect racism and that they had felt disadvantaged because of their ethnicity, including one who felt that their university studies had been severely impacted by indirect racism.

\subsection{The discussions}

Once the results of the questionnaire had been collected, two interviews and one focus group discussion were held via Zoom due to Coronavirus restrictions. Both authors attended all of the discussions, and while it must be admitted that the involvement of teaching staff known to the participants may have limited direct criticism, the contributors agreed that they would be honest and frank in their views, and no reticence was detected by the authors.

The resulting discussions have been collated and grouped by subject area to summarise findings.

\subsubsection{Terminology and ethnic grouping}

All of the participants saw the terms BAME and BME as largely positive, although there was misunderstanding from at least one person as to what the acronym stands for. The general view was that it was being used to bring positive focus to groups of people who had been overlooked. However, two participants did express some reservations, with one feeling that it could lead to deceptive data, and the other feeling that it was restricting focus on distinct backgrounds: 
'People use that category to solve everything...they don't really differentiate.

Black peoples' culture is different to Asian culture. I do want there to be more material based on differences'

Two participants also highlighted problems with combining all Black students under one banner, drawing attention to the differences between African and Caribbean cultures, and within Africa itself. Both acknowledged withdrawing from the university Black Afro-Caribbean Societies because of too much focus on a culture they did not share, and a third participant also questioned the validity of generalised, Black-focused societies:

'Just because we're Black doesn't mean we have to talk about certain things. They will be like, oh, let's talk about this food or this subject, just because we're Black. And it's like, why do we have to have a Black people's society. There's no White Society - because that would just be racist, right? So, I was like, I don't have to join in this society just because I'm Black.'

\subsubsection{Career paths and role models}

Professional design role models did not play any part in our participants' initial desire to follow a creative path, and they were driven instead by an innate desire to be creative. One participant spoke of the lack of visibility of design as a career choice while growing up:

'In Nigeria, the creative is an architect. I told my parents...that I wanted to be a designer, to which my dad said, "What the hell is that?" - as I knew he would say.'

The lack of creative role models - both while growing up and in university teaching materials - did not appear to generate much concern. Some participants said that they had not really been aware of it, while others had registered the lack of Black content but found it understandable 'because maybe there is not that much out there.' Only one participant said that they found the teaching content slightly lacking in cultural variation: 'I had to kind of work a little bit to find artists.'

One participant had attempted to become a role model to two younger Black design students while at university and had been successful in convincing one not to drop out of their course. None of our participants had been exposed to visits from Black alumni while at university, and it was acknowledged that this would have been helpful.

\subsubsection{Student representation and the conversation about race}

Black representation at every committee level in university was considered as a positive step, with one participant questioning the validity of a representative speaking about a subject that they have not experienced. One participant became a senior student union representative as a direct result of their negative experiences as a student: 'I just wanted to turn my sour experience into something good.'

Likewise, all of our participants were enthusiastic about encouraging open conversation about racial issues - though it must be acknowledged that they would have been unlikely to have agreed to take part in this research otherwise - and it was noted that this type of frank conversation is stifled in a higher education environment, with both staff and students loath to open up:

'There is that feeling of holding back because of not seeing people that look like you, so therefore you may feel like they may not understand.'

\subsubsection{Support networks and relationships with lecturers}

Most of the participants had accessed some form of support while at university, for a variety of reasons ranging from financial to psychological. Most spoke of the difficulties of being away from the traditional family support network, the struggle to find an alternative form of support and the stigma of seeking professional help. The idea of specialist Black advisers was briefly discussed, with reference to the BAME mental health services currently being offered at Bristol University [9] and this was felt to be positive in the context of offering choice. All of our participants professed to having a strong Christian faith, and this was often cited as being a strong source of support.

The general feeling was that university support, when sought, had been positive and useful, and that differences in skin colour had caused no issues. However, one participant spoke at length of how seeking 
advice from one lecturer had soured their relationship with the teaching staff and their peers for the remainder of their course, when the lecturer mentioned the issue openly in class:

'It could have come from a good place; however, that wasn't the environment for it, when it was asked in front of other students that I wasn't even friends with but were in the same year, them having to know something that I was deeply struggling with. It sort of robbed a relationship that could have been.'

As a result, the participant - who had previously been a 'social butterfly' - suffered withdrawal, difficulties in forming friendships and confidence issues 'which was something that was never really a feeling of mine until I was put into a space of being a minority.' This also led to a growing estrangement from the teaching staff:

\section{A lot of lecturers didn't even try with me... I'll see them move to a white student- they'll be laughing and stuff like that... I felt for one second there was something up with me. And now looking back... I regret not saying anything.'}

Several participants discussed 'microaggressions' perpetrated by staff. Two examples related to ignorance about the complexities of Black hairstyling, and another stemmed from an assumption that a Malawi student was from Kenya. While these memories were often relayed in a humorous way during the focus groups, they clearly made a lasting negative impression:

\section{'That lack of understanding - although to them it may be humour - it was quite offensive, and it was an ongoing thing.'}

'You don't have to go that extra mile if you don't fully know where this person is from, or you don't really fully know the cultural context. We are all students, we all need to keep on learning, even as a tutor. I would appreciate that even more.'

The proposal of a 'toolkit' to help staff communicate better with students was felt to be a positive idea.

\subsubsection{Group work and assessments}

The participants had all experienced a fair degree of group work while at university and there was a mixed response as to whether there was any disadvantage felt being the only Black member. One participant in particular spoke of their first experience of group work being a defining moment:

\section{I really struggled and that's when I start realising, "ok, there is a clear difference here", whereas before I never saw a difference with colour.'}

This experience led to the participant realising that they felt naturally more comfortable in a group with other Black students, despite making a special effort to try and fit in with white peers, 'and I could never really figure out why that was.'

The issue of anonymised marking was also raised, but it was felt that this would be detrimental in a creative environment, and that teaching staff should take into account how hard students have worked and 'have a little bit of bias.'

\section{COMPLEX ISSUES = VERY SLOW PROGRESS}

The authors' review of university initiatives to reduce the Black awarding gap led to more questions than answers, especially in the context of creative degrees. Anonymised marking, for example, is problematic due to the continuously assessed studio-based nature of creative course work, and there was little support for this from our interviewees. Student engagement with projects focused on addressing barriers to student success is generally good but students are rarely included in policy making and programme development at a high enough level to represent a broad enough voice of those underrepresented in HE. In addition, awarding gap-focused projects are generally underfunded and represent a very small part of services provided by universities. To bring successful change, efforts to close the gap need to be an integral element across all aspects of university life and need to be boughtinto by senior management [10]. Outputs are hard to quantify as data collection is not streamlined and often related to central university reporting. However, it is alarming that the issues discussed at great 
length in the early 2000s by Connor et al. for the UK Government [4] show no sign of improvement nationally. Where progress is being made - such as at Kingston University - it is through relatively simple measures such as student and alumni mentorship and giving academics time to engage with students on a more individual basis [10]. Measures such as these are in direct opposition to the increase in the student-to-staff ratio experienced nationally by UK universities over the last decade [11]. Large student numbers make individual relationship development and student-centred teaching increasingly difficult for staff. Unconscious bias could also easily present as a problem in these large cohort environments as a Black student tutored on a more individual basis than their white peers' challenges parity across the course.

The small student sample interviewed for this paper highlighted how individual each student scenario is and that categorising students under the umbrella terms of BAME or Black, while a positive start, fails to reflect the nuanced approach that is needed. Each story shared was very different, affected by family, culture, community, and different levels of financial hardship. Those with international backgrounds particularly struggled to find their place amongst their peers, consequently leading to isolation, mental health issues and drop in performance. What all the participants had in common was the desire to be better understood and supported by their lecturers and peers, and to discuss the issue openly:

\section{'Just having the conversation in itself is actually really, really great because I feel} like a lot of Black students have really suffered in silence.'

To create truly secure and inclusive futures the black attainment gap needs to urgently become a thing of the past.

\section{REFERENCES}

[1] Universities UK. Universities UK calls for urgent action on racial harassment in higher education. Available: https://www.universitiesuk.ac.uk/news/Pages/uuk-calls-urgent-actionracial-harassment-higher-education-november-2020.aspx [Accessed on 2021, 16 January], (2020, 24 November).

[2] Roberts N. and Bolton P. Educational outcomes of Black pupils and students. UK Government House of Commons Library. Briefing paper no. 09023, 2020.

[3] Khan Y. and Cowell J. Black applicants least likely to be offered PhD places. Available: https://www.bbc.co.uk/news/education-54934953 [Accessed on 2020, 14 December], (2020, 17 November).

[4] Connor H., Tyers C., Modood T., and Hillage J. Why the difference? A closer look at Higher Education minority ethnic students and graduates. UK Government Department for Education and Skills. Research report RR552, 2004

[5] Office for Students. Differences in student outcomes. Available: https://www.officeforstudents.org.uk/data-and-analysis/differences-in-studentoutcomes/ethnicity/ [Accessed on 2021, 26 January], (2019, March).

[6] Office for Students. Continuation and transfer rates. Available: https://www.officeforstudents.org.uk/data-and-analysis/continuation-and-transferrates/continuation-non-continuation-and-transfer-rates/ [Accessed on 2021, 26 January], (2019, April).

[7] UCL Student Success and Progression. BAME Awarding Gap Project-Staff Toolkit 2020. London: University College London. March 2020.

[8] Morris N. The BAME debate: why terminology matters when we're talking about race. Available: https://metro.co.uk/2020/07/07/bame-debate-why-terminology-matters-when-talkingabout-race-12954443/ [Accessed on 2020, 14 December], (2020, 7 July).

[9] BBC News. Black students have a different uni experience. Available: https://www.bbc.co.uk/news/av/uk-england-bristol-52823199 [Accessed on 2021, 5 March], (2020, 28 May).

[10] Warrick Economics \& Development Ltd. Evaluation of the OfS Addressing Barriers to Student Success Programme - Summative Evaluation - Final Report. Available: https://www.w-ecd.com. [Accessed on 2021, 28 February], (2020, July).

[11] Universities UK. Patterns and trends in UK higher education 2018. Available: https://universitiesuk.ac.uk/facts-and-stats/data-and-analysis/Documents/patterns-and-trends-inuk-higher-education-2018.pdf [Accessed on 2021, 4 March], (2018, September). 